\title{
Evidence for a national problem: continued rise in tuberculosis case numbers in urban areas outside London
}

\author{
Michelle E Kruijshaar, ${ }^{1}$ Ibrahim Abubakar, ${ }^{1,2}$ Martin Dedicoat, ${ }^{3}$ Graham H Bothamley, \\ Helen Maguire, ${ }^{5,6}$ Jonathan Moore, ${ }^{1}$ Jonathan Crofts, ${ }^{1}$ Marc Lipman ${ }^{7}$
}

${ }^{1} \mathrm{~TB}$ Section, Respiratory

Diseases Department, Health Protection Agency, Health

Protection Services Colindale, London, UK

2Department of Medicine, Norwich Medical School, University of East Anglia, Norwich, UK

${ }^{3}$ Heart of England Foundation Trust, Birmingham, UK ${ }^{4}$ Homerton University Hospital, London, UK

${ }^{5}$ Health Protection Agency, Health Protection Services Victoria, London, UK ${ }^{6}$ European Centre for Disease Control, Stockholm, Sweden ${ }^{7}$ Centre for Respiratory Medicine, Royal Free Hospital, UCL, London, UK

\section{Correspondence to}

Dr M E Kruijshaar, Health

Protection Services Colindale, 61 Colindale Avenue, London NW9 5EQ, UK; michelle. kruijshaar@hpa.org.uk

Received 25 July 2011 Accepted 23 November 2011 Published Online First 10 January 2012

\begin{abstract}
WHO standards for tuberculosis (TB) control require monitoring and evaluation of TB control programmes. In London, TB rates have stabilised at 44 per 100000 since 2005. In 38 urban areas outside London with TB rates above the national average, these continued to rise after 2004, to 28 per 100000 in 2008 (15\% increase). London has the highest proportion of TB cases in certain risk groups, but these are increasing rapidly outside London. Many TB control efforts focus on the capital, but with rates rising elsewhere in the country, this strategy is likely to fail in the long term.
\end{abstract}

\section{INTRODUCTION}

Unlike most Western countries, the UK has an ongoing increase in tuberculosis (TB). ${ }^{1}$ London accounts for nearly half of all UK TB cases and has high rates of active TB (44 per 100000 in 2008). ${ }^{1}$ Recent initiatives to combat these high rates include raising awareness among ethnic minority groups and increased efforts to find, treat and retain 'hard to reach' patients such as those who are homeless.

Although much attention has focused on London, high rates of $\mathrm{TB}$ are also found in other urban areas such as Birmingham and Leicester. ${ }^{1}$ However, there has been no systematic attempt to determine whether the trends in $\mathrm{TB}$ and characteristics of cases in these areas are similar to those in London, and therefore if these TB control strategies applied in London might also be helpful in other urban areas.

Robust information underpins public health policy. Here we investigate the trends in TB and compare the characteristics of people with $\mathrm{TB}$ in Greater London with those residing in areas outside London where the TB incidence rate is above and below the national average.

\section{METHODS}

TB cases reported to the Enhanced Tuberculosis Surveillance system between 2000 and 2008 in England and Wales were included in the analysis. The Enhanced Tuberculosis Surveillance system collects demographic and clinical information on cases of active TB. These cases are confirmed by a positive culture of Mycobacterium tuberculosis complex or clinically diagnosed, which requires a clinician's judgement that the patient's clinical and/or radiological signs and/or symptoms are compatible with $\mathrm{TB}$, and a decision to treat the patient with a full course of anti-TB treatment.

Average incidence rates of active TB disease for 2006-2008 were calculated for each local government area (local authority) using mid-year estimates provided by the Office for National Statistics. Local areas were grouped into those in Greater London, and those in areas outside London with TB rates above ('outside above') and below ('outside below') the 2006-2008 national average of 14.9 per 100000 .

Trends in the number of cases over time were assessed with the $\chi^{2}$ trend test. Characteristics of cases were tabulated for the three areas.

\section{RESULTS}

\section{TB rates in London and outside}

The national average TB rate in 2006-2008 was 14.9 per 100000 . The overall rate of disease in Greater London was 44.1 per 100000 in this period. Outside London, 38 areas had rates above the national average (box 1), giving a combined rate of 28.0 per 100000 . The remaining 277 'outside below' areas had a rate of 5.3 per 100000

Incidence rates varied substantially in London. Newham and Brent had rates over 100 per 100000 during 2006-2008, Ealing was 70, and a further 14 areas were above 40 per 100000 . Richmond upon Thames, Havering, Bexley, Bromley and Sutton fell below the national average. Outside London, Leicester had the highest rate (70 per 100000$)$, and only three other areas were over 40 per 100000 (Slough, Luton and Birmingham).

\section{Trends}

Between 2000 and 2008 the number of TB cases rose by $28.1 \%$ in London and by $33.4 \%$ in the 38 'outside above' areas (figure 1). Numbers also increased in 'outside below' areas (28.1\%), but rates of disease here were much lower.

Over the entire period, there was no significant difference in the trends in numbers between London and the 'outside above' areas ( $p$ trend $=0.09$ ). However, when the period is split into two phases, case reports are found to have increased more rapidly in London between 2000 and 2004 ( $p=0.001)$. After 2004, however, the rise is predominantly in 'outside above' areas $(p=0.001)$, and to a lesser extent in 'outside below' areas $(p=0.04)$. TB rates show a similar picture, 
Box 1 Local areas outside London with a tuberculosis rate above the national average, England and Wales, 2006-2008

Bedford, Birmingham, Blackburn with Darwen, Bolton, Bradford, City of Bristol, Cambridge, Cardiff, Coventry, Crawley, Derby, Hyndburn, Kirklees, Leeds, Leicester, Luton, Manchester, Middlesbrough, Milton Keynes, Newcastle upon Tyne, Northampton, Nottingham, Oadby and Wigston, Oldham, Oxford, Pendle, Peterborough, Preston, Reading, Rochdale, Rugby, Sandwell, Sheffield, Slough, Walsall, Watford, Woking, Wolverhampton.

stabilising in London after 2005 while continuing to increase in the other two areas (figure 1).

\section{Patient characteristics}

London had the highest proportion of cases that were young adults $(66 \%)$, non-UK born (82\%), and of non-white ethnic group $(87 \%)$. In the 'outside above' areas these percentages were $59 \%, 70 \%$ and $83 \%$, respectively. 'Outside below' areas had the lowest proportion of patients in these groups: $47 \%, 50 \%$ and
$48 \%$. The gender ratio was very similar in all three areas (around 55\%).

London also had the highest proportion of cases presenting with extra-pulmonary disease (47\% compared with $45 \%$ and $35 \%$ in 'outside above' and 'outside below' areas, respectively), but a smaller proportion of pulmonary cases were sputum smear positive (54\% compared with $57 \%$ and $59 \%$, respectively). In London $10 \%$ of cases were resistant to one or more first-line drugs compared with $6 \%$ in the other areas. The same proportion of cases were culture confirmed in the three areas (55\%).

While in London the proportion of TB cases that were non-UK born remained relatively stable between 2000 and 2008, this increased from $63 \%$ to $72 \%$ in 'outside above' areas and from $35 \%$ to $58 \%$ in 'outside below' areas. This was reflected in a rise in the black African ethnic group in 'outside above' areas and in black African, and Indian, Pakistani and Bangladeshi ethnic groups in 'outside below' areas. In all three areas, the proportion of cases that were culture confirmed showed a similar fluctuation over time, increasing initially but then stabilising.

\section{DISCUSSION}

This is the first study to show that from 2005 onwards the incidence of TB in London has stabilised, while outside London, especially in urban areas, it is increasing. We believe that this is a real effect as we found similar levels of $\mathrm{TB}$ culture

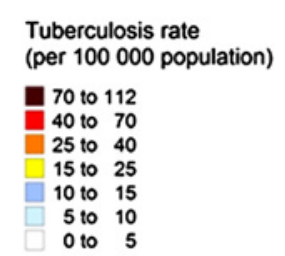

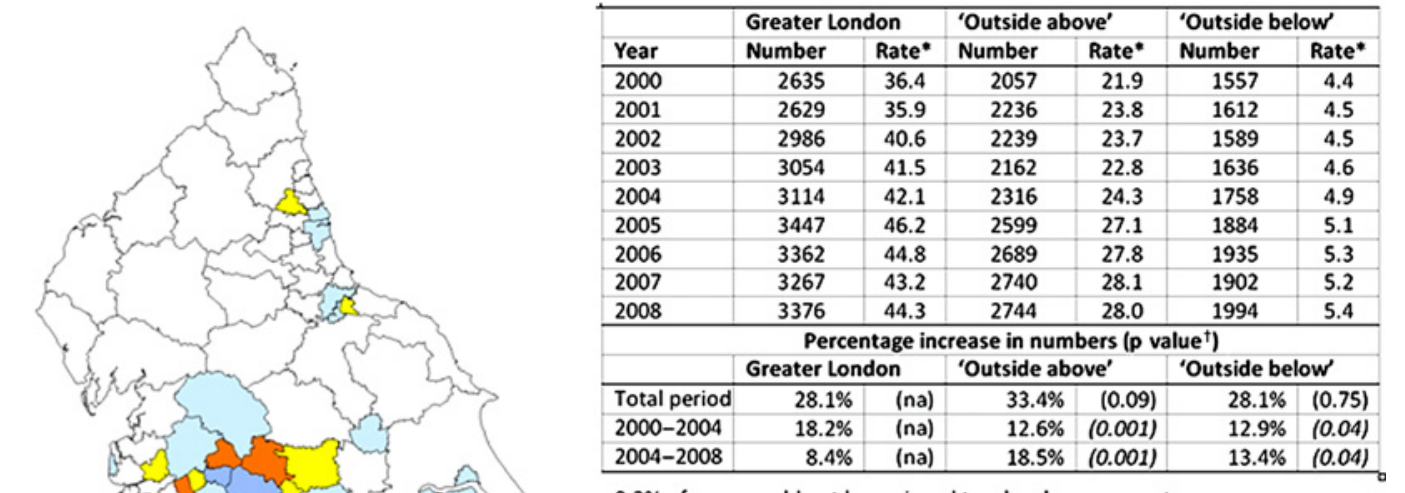

$0.2 \%$ of cases could not be assigned to a local government area.

- Rate per 100000 population

$+\chi^{2}$ trend test for rise in numbers compared to Greater London.

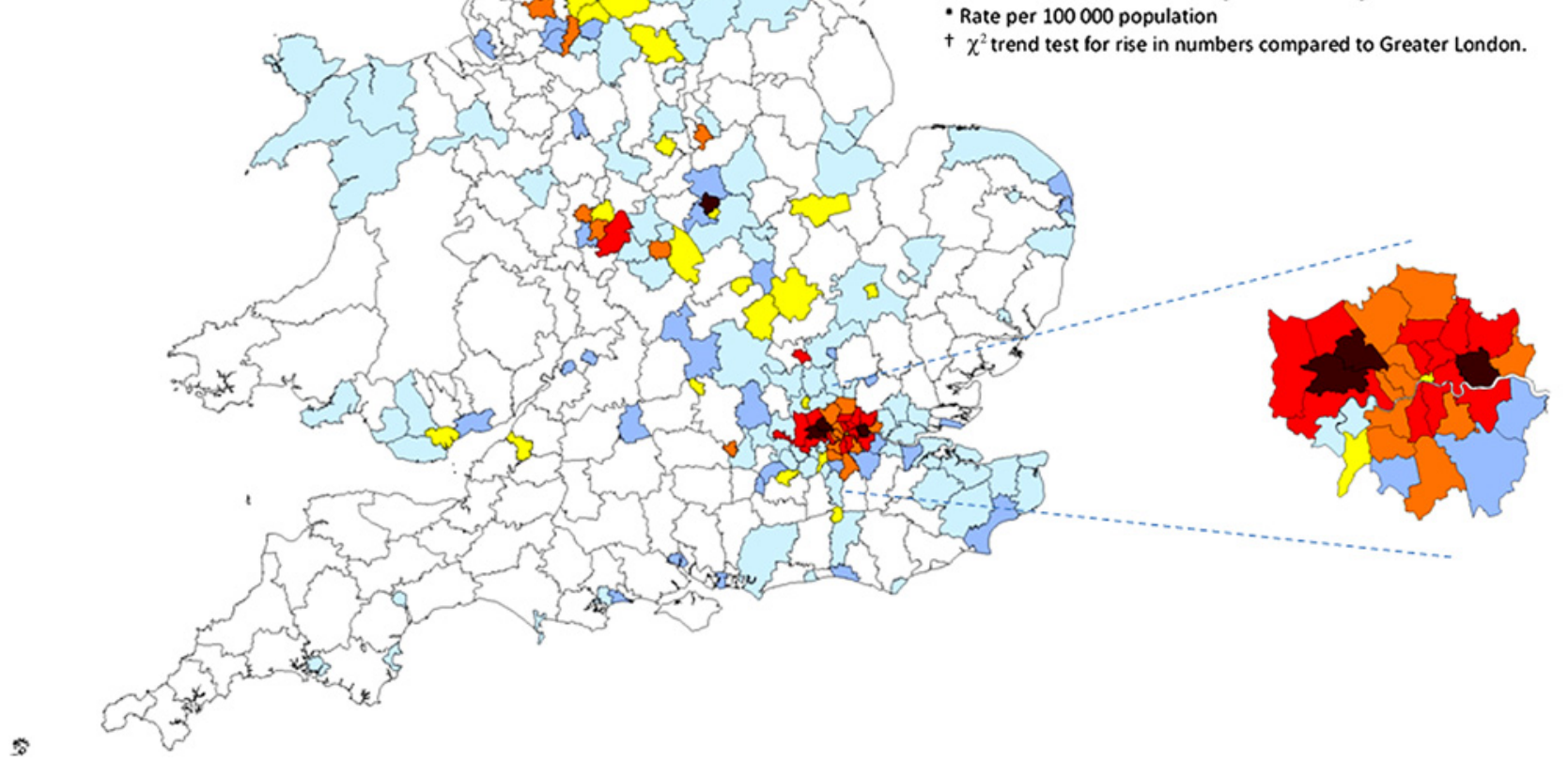

Figure 1 Three-year average rate of tuberculosis (TB) by local authority, and change in the number of TB cases reported in London and areas outside London, England and Wales 2000-2008. 
confirmation, and trends therein over time, in the three areas. Therefore, our results are unlikely to represent an artefactual increase in clinical diagnosis (without culture confirmation) outside of London.

The substantial rise in the proportion of non-UK born TB cases outside London suggests that shifts in migration patterns-with more migrants now locating to these urban areas-could be driving the observed temporal trends. It is also possible that the implementation of awareness-raising activities and increased efforts to find, treat and retain patients in London have played a role in the stabilisation observed in the capital. Information on trends in the underlying population of local areas is required to investigate adequately the effect of migration. Labour Force Survey figures are not helpful here as these become less reliable when applied to smaller, local areas.

Groups at high risk of $\mathrm{TB}$, such as immigrants, those coinfected with HIV and socially deprived populations tend to be over-represented in large cities. This explains the higher rates in urban areas. ${ }^{2}$ Cases in the urban 'outside above' areas were often non-UK born young adults and from ethnic minority groups, although the proportions were smaller than in London. Data on HIV co-infection, deprivation, homelessness and drug use were not available for analysis. These data were gathered in 2009 and examination suggests that, like London, homelessness and drug use are common among people with $\mathrm{TB}$ in, for example, the Birmingham metropolitan area. We suggest that strategies shown to be successful in London should be applied to other parts of the country with a similar TB demographic.

New immigrant screening is often recommended. However, most cases of TB occur several years after arrival in the UK. ${ }^{1}$ This is unlikely, therefore, to be an efficient or cost-effective approach. What may be more successful is the use of community-based campaigns which encourage prompt, appropriate health-seeking behaviour in populations at risk of TB who develop symptoms consistent with disease. In addition, the use of primary care-centred new registration health checks which include information on, and assessment for, TB would be helpful. ${ }^{4}$

Variability in trends and case characteristics clearly exist within each of the three groups. For example, despite London rates stabilising since 2005, those in Newham, which has the highest incidence of all boroughs, have continued to rise. Specific migrant and ethnic minority groups also tend to congregate in particular cities, and so changes in migration from certain countries will preferentially affect certain locations. Thus a more detailed local TB analysis is needed to inform public health action within a future clinical commissioning group or GP cluster.

\section{CONCLUSION}

Since 2005 rates of TB have stabilised in London and increased in other parts of England and Wales, especially in urban areas. In these areas there are specific and changing demographic and clinical characteristics of $\mathrm{TB}$, including a younger age distribution and an increasing proportion of non-UK born cases. Local and national urban control policies need to reflect these findings. Focusing TB control efforts on the capital city, ignoring other major conurbations, is a strategy which is likely to fail in the long term because rates will rise in other parts of the country.

Acknowledgements We would like to thank everyone who provided information on tuberculosis cases in England and Wales and to acknowledge David Quinn for his work in preparing the Enhanced Tuberculosis Surveillance dataset.

\section{Competing interests None.}

Ethical approval This study was carried out with national surveillance data. The Health Protection Agency has Patient Information Advisory Group approval to hold and analyse national surveillance data for public health purposes under Section 60 of the Health and Social Care Act 2001.

Provenance and peer review Not commissioned; externally peer reviewed

\section{REFERENCES}

1. Anderson L, Moore J, Kruijshaar M, et al. Tuberculosis in the UK: Annual Report on Tuberculosis Surveillance in the UK, 2010. London: Health Protection Agency, 2010.

2. de Vries G, van Hest NA, Baars HW, et al. Factors associated with the high tuberculosis case rate in an urban area. Int J Tuberc Lung Dis 2010;14:859-65.

3. Hayward AC, Darton T, Van Tam JN, et al. Epidemiology and control of tuberculosis in Western European cities. Int J Tuberc Lung Dis 2003;7:751-7.

4. Griffiths C, Sturdy P, Brewin P, et al. Educational outreach to promote screening for tuberculosis in primary care: a cluster randomised controlled trial. Lancet 2007;369:1528-34. 